\title{
ON A CHARACTERISTIC PROPERTY OF ARNOUX-RAUZY SEQUENCES
}

\author{
JACQUes Justin $^{1}$ AND GiUseppe PiRILlO ${ }^{2}$
}

\begin{abstract}
Here we give a characterization of Arnoux-Rauzy sequences by the way of the lexicographic orderings of their alphabet.
\end{abstract}

Mathematics Subject Classification. 68R15.

\section{INTRODUCTION}

Let $s$ be a standard Sturmian infinite word on $A=\{a, b\}$. If we lexicographically order $A^{*}$ by $a<b$ then, for any non-negative integer $n$, the prefix of length $n$ of $a s$ is the smallest factor of length $n$ of $s$ and the prefix of length $n$ of $b s$ is the greatest factor of length $n$ of $s$, see Borel and Laubie [3] and Exercise 2.2.13 in [7]. The converse of this property is true also as was remarked and proved by the second author [8].

Here we extend the property and its converse to Arnoux-Rauzy sequences on finite alphabets $[1,9]$ (or strict Episturmian words in the terminology of $[4,6]$ ), which generalize Sturmian words. This is:

Theorem 0.1. For any infinite word s on a finite alphabet $A$ the following properties are equivalent

i) $s$ is a standard Arnoux-Rauzy sequence;

ii) for any $x \in A$ if $<$ is a lexicographic order of $A^{*}$ satisfying $x=\min A$, then for any positive integer $n$ the prefix of $x$ s of length $n$ is the smallest among the factors of $s$ of length $n$.

Keywords and phrases: Lexicographic order, Arnoux-Rauzy sequence, Episturmian word, Sturmian word.

${ }^{1}$ LIAFA, Université Paris VII, Case 7014, 2 place Jussieu, 75251 Paris Cedex 05, France; e-mail: justin@liafa.jussieu.fr

2 IAMI, NCR, Viale Morgagni 67/a, 50134 Firenze, Italy;

e-mail: pirillo@udini.math.unifi.it 
This may have some interest as many properties of Sturmian words extend to Arnoux-Rauzy sequences but few are characteristic.

Remark. In the formulation of the theorem we do not consider max $A$ nor the greatest factor of each length. Indeed, for any two words $u, v$ of the same length, $u>v$ is equivalent to $v \ll u$ where $\ll$ is the lexicographic order "opposite" to $<$.

\section{Preliminaries}

Let $A$ be a finite alphabet $|A|=k, A^{*}$ (resp. $A^{\omega}$ ) denotes the set of all finite (resp. infinite) words on $A$. The common terminology for words can be found in [7] for instance.

For $s \in A^{\omega}, s_{n}$ will denote the prefix of $s$ of length $n$. The set of the factors of $s$ of length $n$ is $F_{n}(s)$ and the set of all factors of $s$ is $F(s)=\bigcup_{n>0} F_{n}(s)$. A factor $u$ of $s$ is right- (resp. left-) special if, for at least two letters $x \neq y, u x, u y \in F(s)$ (resp. $x u, y u \in F(s)$ ). An infinite word $s$ is recurrent if each of its factors occurs at least two (hence infinitely many) times in it.

The reversal of a word $u=x_{1} x_{2} \cdots x_{n}, x_{i} \in A$ is $\tilde{u}=x_{n} \cdots x_{2} x_{1}$.

An Arnoux-Rauzy sequence on $A$ is a recurrent infinite word $s \in A^{\omega}$ such that for any non-negative integer $n, s$ has exactly one right-special factor, $u_{n}$ say, of length $n$ with $u_{n} x \in F(s)$ for all $x \in A$, and exactly one left-special factor, $v_{n}$ say, such that $y v_{n} \in F(s)$ for all $y \in A[1,9]$. These sequences are the Sturmian words when $|A|=2$. They were studied in [1] for $|A|=3$ with a hint of generalization to any finite alphabet. In their paper the authors assumed the infinite word to be uniformly recurrent (or minimal in the terminology of dynamical systems used there), but indeed the assumption of recurrence is sufficient and leads to exactly the same infinite words.

An Arnoux-Rauzy sequence is standard (or characteristic) if all its prefixes are left-special.

In [4-6] a slightly more general kind of words is studied under the name of "Episturmian words" and it is shown that Arnoux-Rauzy sequences on $k$ letters are exactly the $k$-strict Episturmian words. Episturmian words which are not strict correspond to some "degenerate cases", for instance when $k=2$ they are the so called "periodic Sturmian words".

\section{Proof of the TheOREM}

Proof. $i) \Rightarrow i i)$. Given the lexicographic order $<$ with $x=\min A$ as in the theorem, if $i i)$ is false let $n$ be minimal such that $x s_{n-1}$ is not minimal in $F_{n}(s)$. Clearly $n>1$. Then $x s_{n-2}$ is minimal in $F_{n-1}$. Let $s_{n-1}=s_{n-2} y, y \in A$. As $x s_{n-2} y$ is not minimal in $F_{n}(s)$, there exists a letter $z \neq y$ such that $x s_{n-2} z$ $\in F_{n}(s)$ and that $x s_{n-2} z<x s_{n-2} y$, whence $z<y$. Thus $x s_{n-2}$ is right-special. Thus as $F(s)$ is closed under reversal $[4,6,9], \widetilde{s_{n-2} x}$ is left-special, hence it is 
$s_{n-2} y$ by the definition of Arnoux-Rauzy sequences. Thus $x=y$, whence $z<x$, a contradiction. Consequently, for all $n, x s_{n}=\min F(s)$.

$i i) \Rightarrow i)$. As as has its prefixes in $F(s)$ for all $a \in A$, any prefix $u$ of $s$ is left-special and satisfies $A u \subset F(s)$. Suppose that, for some positive integer $n, s$ has at least two left-special factors of length $n$, its prefix $s_{n}$ and another one $v$ and suppose $n$ minimal with this property. Set $s_{n}=s_{n-1} x, v=v^{\prime} y, x, y \in A$. Then $s_{n-1}$ and $v^{\prime}$ are left-special, hence $v^{\prime}=s_{n-1}$ and $v=s_{n-1} y, y \neq x$. Then there exist letters, $z \neq t, r \neq m$ such that $z s_{n}, t s_{n}, r v, m v \in F_{n+1}(s)$.

Suppose for instance $r \neq x$ (otherwise, $m \neq x$ ) and consider a lexicographic order such that $r=\min A$ and $y<x$. Then $r v=r s_{n-1} y<r s_{n-1} x=r s_{n}$, thus $r s_{n}$ is not minimal in $F_{n+1}$, a contradiction.

Thus, for all $n, s$ has exactly one left-special factor of length $n$ and then $s$ has for all $n$ at least one right-special factor of length $n$. Now suppose $s$ has, for some $n$, two right-special factors of length $n, u$ and $v$ say, and let $n$ be minimal with this property. Then we have $u=x w, v=y w, x \neq y \in A$ where $w$ is the unique right-special factor of length $n-1$. Clearly $w$ is left-special and $w=s_{n-1}$. We then have $s_{n}=w a$ for some letter $a$ whence, as $A s_{n} \subset F(s)$, xwa, ywa $\in F_{n+1}(s)$.

As $u, v$ are right-special, there exist letters, $t \neq a, m \neq a$ such that $x w t$, ywm $\in F(s)$. Suppose for instance $y \neq a$. Consider a lexicographic order $<$ such that $y=\min A$ and $m<a$, we get ywa $>y w m$, thus $y s_{n}$ is not minimal in $F_{n+1}(s)$, a contradiction.

Thus $s$ has for all $n$ exactly one right-special factor $u_{n}$ of length $n$ and as $\left|F_{n+1}(s)\right|-\left|F_{n}(s)\right|=|A|-1, u_{n} A \subset F(s)$.

As clearly $s$ is recurrent it satisfies the definition of Arnoux-Rauzy sequences and as its prefixes are left-special it is a standard one.

Added in proof. The result of [8] appeared under other form in S. Gan, Sturmian sequences and the lexicographic world, Proc. Amer. Math. Soc. 129 (2001) 14531457. It is also related to a common work of Zamboni (private communication), Kruger, Schmeling and Winkler.

\section{REFERENCES}

[1] P. Arnoux and G. Rauzy, Représentation géométrique de suites de complexité $2 n+1$. Bull. Soc. Math. France 119 (1991) 199-215.

[2] J. Berstel, Recent results in Sturmian words, in Developments in Language Theory II, edited by J. Dassow, G. Rozenberg and A. Salomaa. World Scientific (1996) 13-24.

[3] J.-P. Borel and F. Laubie, Quelques mots sur la droite projective réelle. J. Théor. Nombres Bordeaux 5 (1993) 123-137.

[4] X. Droubay, J. Justin and G. Pirillo, Episturmian words and some constructions of de Luca and Rauzy. Theoret. Comput. Sci. 255 (2001) 539-553. 
[5] J. Justin, On a paper by Castelli, Mignosi, Restivo. RAIRO: Theoret. Informatics Appl. 34 (2000) 373-377.

[6] J. Justin and G. Pirillo, Episturmian words and Episturmian morphisms. Theoret. Comput. Sci. 276 (2002) 281-313.

[7] M. Lothaire, Algebraic Combinatorics on Words. Cambridge University Press (2002).

[8] G. Pirillo, Characterization of infinite Surmian words (in preparation).

[9] R.N. Risley and L.Q. Zamboni, A generalization of Sturmian sequences, combinatorial structure and transcendence. Acta Arithmetica 95 (2000) 167-184.

Communicated by Ch. Choffrut.

Received April, 2002. Accepted November, 2002. 